\title{
Supervised probabilistic failure prediction of tuned mass damper-equipped high steel frames using machine learning methods
}

https://doi.org/10.2478/sgem-2019-0043

received May 28, 2019; accepted January 21, 2020.

\begin{abstract}
In this study, firstly, the behavior of a high steel frame equipped with tuned mass damper (TMD) due to several seismic records is investigated considering the structural and seismic uncertainties. Then, machine learning methods including artificial neural networks (ANN), decision tree (DT), Naïve Bayes (NB) and support vector machines (SVM) are used to predict the behavior of the structure. Results showed that among the machine learning models, SVM with Gaussian kernel has better performance since it is capable of predicting the drift of stories and the failure probability with $R^{2}$ value equal to 0.99. Furthermore, results of feature selection algorithms revealed that when using TMD in high steel structures, seismic uncertainties have greater influences on drift of stories in comparison with structural uncertainties. Findings of this study can be used in design and probabilistic analysis of high steel frames equipped with TMDs.
\end{abstract}

Keywords: Failure analysis; supervised machine learning; feature selection; tuned mass damper.

\section{Introduction}

Mass damper systems consist of three components: internal mass, internal spring and damper, which are dynamically placed with a degree of freedom above the structure. When the structure vibrates, the system also begins to vibrate with a phase opposite to the vibrating

\footnotetext{
*Corresponding author: Farshid Farrokhi, Department of Civil Engineering, Noor Branch, Islamic Azad University, Iran, E-mail: farrokhi_farshid@yahoo.com

Sepideh Rahimi, Department of Civil Engineering, Noor Branch, Islamic Azad University, Iran
}

frequency, and its inertial forces will deplete the structure vibrations (Soto and Adeli, 2013).

Hundreds of research studies have been carried out on mass dampers (Marano et al., 2010; Giaralis and Taflanidis, 2018; Wang et al., 2019). Most of these studies are related to the parametric study of the effect of the damper on the behavior of the structure, or the determination of the location and characteristics of the optimal mass damper to achieve the maximum reduction in seismic response.

Bekdaş and Nigdeli (2011) have proposed the optimum parameters of tuned mass dampers (TMD) under seismic excitations. Harmony search (HS), a metaheuristic optimization method, which has been successfully applied for several engineering problems, is revised for tuning passive mass dampers. In another study, Chey et al. (2010) investigated the seismic performance attributes of multi-story passive and semi-active tuned mass damper (PTMD and SATMD) building systems for 12-story moment resisting frames modelled as " $10+2$ " stories and " $8+4$ " stories. Segmented upper portion of the stories are isolated as a tuned mass, and a passive viscous damper or semiactive resettable device is adopted as energy dissipation strategy. Results showed that large SATMD systems can effectively manage seismic response for multi-degree-of freedom systems across a broad range of ground motions in comparison to passive solutions.

Mohebbi et al. (2013) proposed genetic algorithm (GA) for designing optimal multiple tuned mass damper (MTMD) to mitigate the seismic response of structures based on defining an optimization problem, which considers the parameters of tuned mass damper (TMD) as variables and minimization of maximum structural response as an objective function.

By investigating previous studies in the field of TMD, only a few probabilistic studies have been carried out in this area. One of these studies is reported by Debbarma and Debnath (2013), where they have investigated the optimal mass-damper design due to the uncertainties of damper characteristics. Their research included the optimization 
of frequency and TMD damping characteristics with respect to the uncertain parameters of the system. In order to find the optimal values of TMD parameters based on the rules of random vibration, the maximum displacement of a three-story structure is considered as the target.

Giaralis and Taflanidis (2018) considered an optimum tuned mass-damper-inerter (TMDI) design framework accommodating the mass-amplification and highermodes-damping, while accounting for parametric uncertainty to the host structure properties, modelled as a linear multi degree of freedom system. TMDI couples the classical TMD with an inerter, a two-terminal device resisting the relative acceleration of its terminals in judicial topologies. Although they showed that the TMDI achieves enhanced structural performance and robustness to building and excitation uncertainties compared to same mass/weight TMDs, further probabilistic analysis is required to reveal the actual performance of TMDIs.

Wang et al. (2019) introduced a self-adjustable variable pendulum tuned mass damper (SAVP-TMD). On the basis of the acceleration ratio between TMD and primary structure, they showed that SAVP-TMD can retune itself by varying the length of the pendulum according to the improved acceleration ratio-based adjustment algorithm. However, they verified their proposed system on a five-story structure and not on a tall frame.

In order to achieve the most acceptable accuracy of modelling especially for high steel structures, the effects of uncertainties should be considered efficiently in the calculations. This uncertainty ultimately leads to an uncertainty in the evaluation of seismic response of the structure (Deierlein et al., 2010). In order to evaluate the behavior of structures in recent years, statistical and probabilistic methods have been used to quantify the possibility of failure (Farrokhi and Rahini, 2017).

Due to the recent developments in inexpensive computers and processing equipment, machine learning has become an applicable solution in pattern recognition and nonlinear modelling of big data (Michalski et al., 2013; Massah and Asefpour Vakilian, 2019). Statistical regression models provide a mathematical equation to calculate the dependent variable of a sample by having the input features. Machine learning methods can learn a database including hundreds of input features and corresponding dependent variables (in this situation, they are called "targets") of a number of samples to predict the target of a new sample having its input features (Ahmad et al., 2014). Hundreds of machine learning algorithms have been developed for engineering problems, which can be implemented in programming environments such as MATLAB, Python, and so on.
The objective of this study was: a) to investigate the behavior of a high steel frame equipped with tuned mass damper (TMD) due to several seismic records considering the structural and seismic uncertainties, and b) to study the performance of different supervised machine learning algorithms in prediction of the behavior of the structure.

\section{Materials and methods}

\subsection{Structure modelling}

A two-dimensional 20-story steel frame consisting of 4 five-meter openings was modelled in this study (Figure 1). The regulations used for steel construction standards, loading and seismic rules were based on Section 10 of National Building Regulations of Iran, Section 6 of the National Building Regulations of Iran, and the 2800 Regulations (third edition), respectively. The 20-story frame was designed using the SAP2000 program and the sections were logically selected. The values of dead and dynamic loads of stories and roofs are presented in Table 1. The characteristics of the structure are shown in Table 2. Mathematical modelling and analysis of similar tall structures towards different loadings and ground motions can be found in literature (Krishnan, 2007; Muto and Krishnan, 2011).

The design is carried out in several steps, so selecting the sections was close to the optimal conditions in terms of the amount of tensions and lateral displacement of the structure. Therefore, the effects of each type of components on the overall behavior of the structure can be properly and comprehensively analyzed. The elements used in the structure model were beams and columns; for columns, rectangular cans made of steel sheets, and for beams, IPEs were considered. It should be noted that the compression of all sections used in the structure such as cans was ensured.

\subsection{TMD modelling}

In order to investigate the effectiveness of the vibrationabsorbing devices on steel moment-resisting frames under seismic loads, a TMD was considered on the top story of the structure during the simulation. TMD was added to the primary structure to absorb the energy of the applied excitation achieving a reduction in the damage of the structural elements and improve the performance of the structure (Soto et al., 2013). 


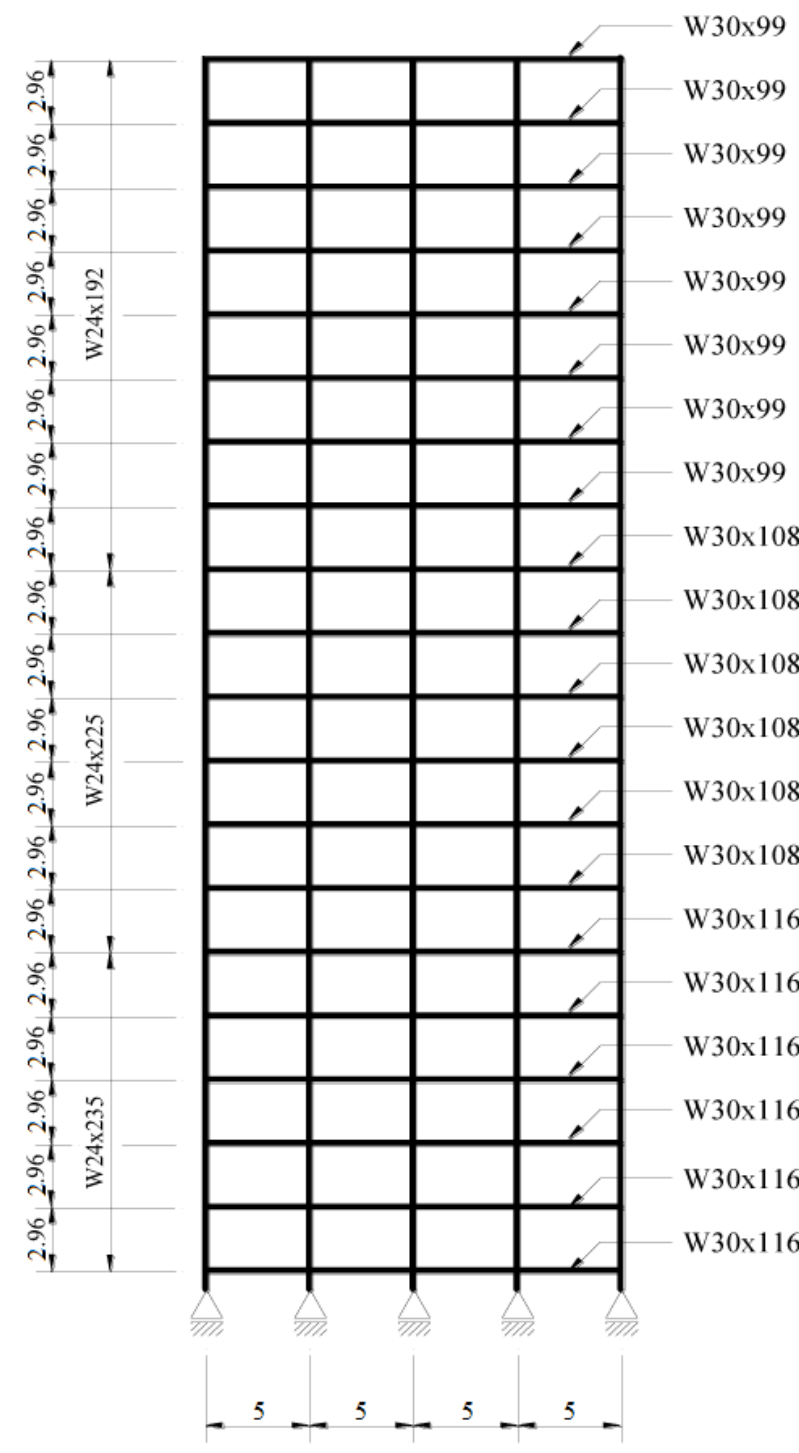

Figure 1: Studied 20-story steel structure.

Since the mass ratio, which is defined as the ratio of the TMD mass to mass of the structure, is generally chosen between $1 \%$ and $10 \%$ in designing TMDs (Pozos-Estrada and Hong, 2015), TMD mass was considered equal to $2 \%$ of the total mass of the studied frame. Structural stiffness was also considered in a way so that the period of TMD was similar with the first mode of the structure (Vickery et al., 2001). The characteristic parameters of TMD was calculated based on the method proposed in Sadek et al. (1997). In this method, for a given mass ratio, the stiffness of the TMD spring $(k)$ and the viscous damping coefficient of the damper $(c)$ are calculated using Equations (1) and (2), respectively:

$$
k=m \omega_{T M D}^{2}
$$

Table 1: Dead and dynamic loads of stories and roofs.

\begin{tabular}{lll}
\hline & Dead load $\left(\mathrm{kg} \cdot \mathrm{m}^{-2}\right)$ & Dynamic load $\left(\mathrm{kg} \cdot \mathrm{m}^{-2}\right)$ \\
\hline Stories & 1250 & 500 \\
Roof & 1125 & 375 \\
\hline
\end{tabular}

Table 2: Characteristics of the structure.

\begin{tabular}{ll}
\hline Feature & Value \\
\hline Height of structure $(\mathrm{m})$ & 60 \\
Period of structure (s) & 1.7247 \\
Coefficient of reflection & 0.9023 \\
Coefficient of importance & 1 \\
Coefficient of behavior & 7.5 \\
Acceleration scheme & 0.35 \\
Coefficient of earthquake & 0.0421 \\
\hline
\end{tabular}

$$
c=2 m \xi \omega_{T M D}
$$

where $m$ in the mass of TMD, $\omega T M D$ is the circular frequency of TMD, and $\xi$ is the damping ratio of TMD and is calculated using Equation (3):

$$
\xi=\Phi\left[\frac{\beta}{1+\mu}+\sqrt{\frac{\mu}{1+\mu}}\right]
$$

where $\Phi$ is the component of the first mode of TMD, $\beta$ is the damping ratio of the structure, and $\mu$ is the mass ratio (Sadek et al., 1997).

\subsection{Incremental dynamic analysis algorithm}

Nonlinear dynamic analysis was used to achieve the statistical parameters in failure capacity. This analysis is usually performed to measure the midrange capacities and standard deviation of failure ratios. Gravitational loads for analysis are different from designing gravitational loads and are determined by the summation of dead weight and $20 \%$ of the dynamic weight. Nonlinear dynamic response of the considered frame for a set of previously reported earthquakes using incremental dynamic analysis (IDA) is calculated to the extent that the structure will be unstable or reaches the desired performance level. To carry out this kind of analysis, the structure should be excited by an earthquake record. One of the usual ways is to initially excite the structure under an earthquake 
with a maximum acceleration of $0.1 \mathrm{~g}\left(\mathrm{~g}=9.8 \mathrm{~m} . \mathrm{s}^{2}\right)$ and add $0.1 \mathrm{~g}$ to the maximum acceleration at each step. Peak ground acceleration (PGA) was changed from 1 to $10 \mathrm{~m} \cdot \mathrm{s}^{-2}$ to investigate the seismic uncertainties, whilst material yield strength was changed between 222 and $260 \mathrm{MPa}$ with $2 \mathrm{MPa}$ steps during the experiments to study the effects of structural uncertainties. The reason for such a range is that the yield strength of the steel wide flange beam, which was selected for the structure simulation, is ca. $240 \mathrm{MPa}$.

The flowchart of IDA analysis carried out in this study is shown in Figure 2. For analysis, OpenSees and MATLAB software have been used in a loop. Initially, using a code written in MATLAB, a sub-procedure was created in the $\mathrm{Tcl}$ environment containing the earthquake record information. Then, considering that the created model in OpenSees should be modified for each IDA, modification was done automatically using MATLAB using iterative loops.

In order to facilitate the construction of sections, two sub-programs were used in the Tcl environment, one of which for constructing the columns and the other one for I-beams. The code written in OpenSees performed an IDA analysis on the structure at each run, and wrote out the output in text files. Upon completion of the analysis, the outputs were processed again using MATLAB and IDA results were plotted.

\subsection{Machine learning modelling}

In this study, machine learning was used to predict story drifts in different PGAs and uncertainties in the material yield strength of the structure. In this situation, story drift was considered as the target, while seismic and structural uncertainties were the input features.

By having the database, decision tree (DT), random forest (RF), Levenberg-Marquardt back-propagation artificial neural networks (ANN), support vector machine (SVM), least-square support vector machine (LSSVM) and Naïve Bayes (NB) machine learning models were used for the prediction of story drifts.

Different types of DT modeling including iterative dichotomiser 3 (ID3), statistical model (C4.5), classification and regression tree (CART), chi-squared automatic interaction detection (CHAID) and multivariate adaptive regression splines (MARS) were used for nitrate determination (Singh and Gupta, 2014).

One of the important parameters of the artificial neural network that significantly affects the performance of this model is the network architecture, that is, the size

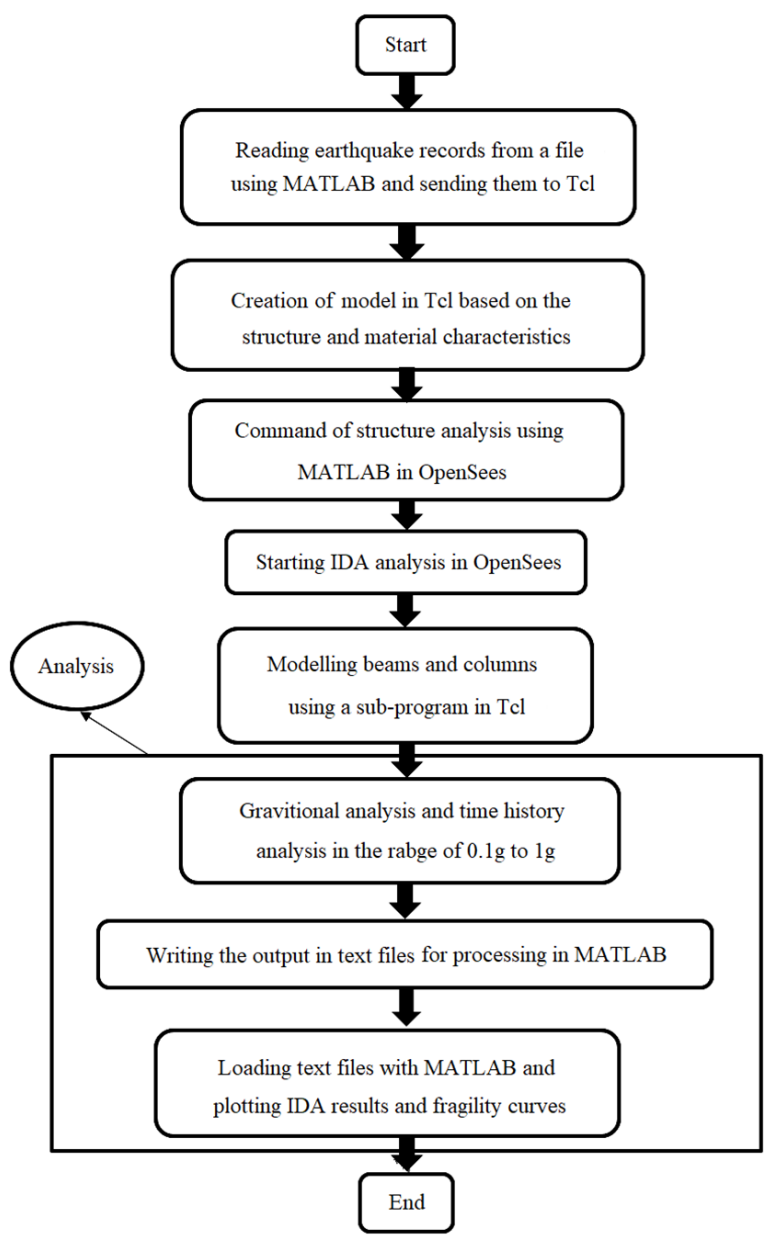

Figure 2: Incremental dynamic analysis algorithm.

of network hidden layer (Asefpour Vakilian and Massah, 2018). The size of the hidden layer varied from 2 to 10 with 2 nodes step to obtain the optimum network.

SVM and LSSVM models have two parameters including kernel type and kernel parameter that affect the performance of the model (Basak et al., 2007). Three kernel types including polynomial $\left(f=(y x x o)^{3}\right)$, radial basis function $\left(f=\exp \left(-y(x-x o)^{2}\right)\right)$ and sigmoid $(f=$ $\tanh (y x x o))$ were considered for modeling, where $f$ is the kernel function, $y$ is the kernel parameter, $x$ is a train or test sample in the modeling hyperplane, and $x o$ is the origin point in the hyperplane (Basak et al., 2007).

The algorithms of the machine learning methods were implemented using a code written in MATLAB R2016b programming environment (Mathworks, Massachusetts, United States).

To investigate the performance of the machine learning methods, 5-fold cross-validation was used for training and testing. The first step in machine learning modeling is data normalization. Targets were normalized 
using Equation (4) to make it possible to compare the error parameters of the models. Equation (4) maps all values for each feature and the target between 0 and 1 .

$$
x_{n}=\frac{x_{o}-x_{\min }}{x_{\max }-x_{\min }}
$$

where $x n$ is a sample's target value after normalization, $x o$ is the raw target value, and $x_{\min }$ and $x_{\text {max }}$ are the lowest and highest targets in all samples, respectively. The performance of the models was evaluated based on the mean square error (MSE) and the coefficient of determination $\left(R^{2}\right)$ using Equations (5) and (6), respectively:

$$
\begin{gathered}
\mathrm{MSE}=\frac{1}{n} \sum_{i=1}^{n}\left(x_{\mathrm{p}}-x_{\mathrm{m}}\right)^{2} \\
R^{2}=1-\frac{\sum_{i=1}^{n}\left(x_{\mathrm{p}}-x_{\mathrm{m}}\right)^{2}}{\sum_{i=1}^{n}\left(x_{\mathrm{m}}-\bar{x}_{\mathrm{m}}\right)^{2}}
\end{gathered}
$$

where $x_{\mathrm{m}}$ is the measured value of story drift in the dataset and $x p$ is the predicted story drift using machine learning. The lower MSE and higher $R^{2}$, the better the performance of the machine learning model is.

\subsection{Feature selection algorithms}

Feature selection (i.e., variable selection) is generally one of the fundamental problems in machine learning and pattern recognition. In recent years, according to new emergences in computer applications, datasets with thousands of features have been created (Liu et al., 2002). However, most of the features in these datasets are irrelevant or redundant, which result in low efficiency and even over-fitting of the learning algorithms (Sun et al., 2012). Therefore, feature selection has become one of the most active research areas to solve this problem. The essential idea of feature selection is to detect the irrelevant and redundant features from data sets as many as possible. Feature selection in machine learning aims to find a good feature subset which produces higher classification accuracy (Guyon and Elisseeff, 2003).

Several approaches are employed in feature selection, such as genetic algorithm (Huang et al., 2007), simulated annealing (Lin et al., 2008), SVM (Moustakidis et al., 2010) and boosting method (Liu et al., 2009), which they can typically fall into three categories: embedded, wrapper and filter methods. In this study, Relieff algorithm, t-test, sequential feature selection (SFS) and class separability criteria (CSC) were used to score the effects of structural and seismic uncertainties on the prediction of story drifts during seismic loadings.

\subsection{Seismic records}

In this study, several earthquake records with characteristics shown in Table 3 were selected for IDA analysis in order to create a dataset that was used later in machine learning and feature selection.

\section{Results and discussion}

\subsection{IDA results}

After processing the data obtained from OpenSees in MATLAB using the flowchart shown in Figure 2, the IDA data were extracted, as shown in Figure 3 for the first, fifth, tenth, fifteenth, and twentieth stories of a 20-story structure. Figure 3 illustrates the IDA plots for the Northridge event in Castaic - Old Ridge Route station. The IDA results for other seismic events and stations were obtained similarly for creating the dataset.

The drift of each story is considered as the relative displacement with regard to the upper and lower stories, except the first story that I reported with regard to the upper story. Figure 3 shows that by increasing the PGA, relative displacement of the stories has increased in general. However, its increasing trend is not similar for all the stories. When not using TMD in the structure, for the tenth story of the structure, relative displacement was initially increased with increasing the PGA, but for the largest PGA, this relative displacement decreased. This can be attributed to the natural period of the structure, earthquakes and their impact. It is also observed that when the TMD has not been used in the structure, the largest relative displacement belongs to the tenth story ( $46 \%$ for $\left.\mathrm{PGA}=9 \mathrm{~m} \cdot \mathrm{s}^{-2}\right)$, followed by the fifteenth story $(20 \%$ for $\mathrm{PGA}=10 \mathrm{~m} \cdot \mathrm{s}^{-2}$. In severe seismic loads, the input energy to the structure is increased and a bigger part of the energy is dissipated due to the creating plastic deformations.

These deformations are due to the formation of local plastic joints in the structure, which itself increases the energy dissipation in the system (Kappos and 
Table 3: Studied earthquake records and their characteristics.

\begin{tabular}{|c|c|c|c|c|}
\hline Event & Year & Magnitude & Radius (km) & Station \\
\hline Cape Mendocino & 1992 & 7.1 & 23.6 & Fortuna - Fortuna Blvd \\
\hline Cape Mendocino & 1992 & 7.1 & 18.5 & Rio Dell Overpass - FF \\
\hline Duzce & 1999 & 7.1 & 15.6 & Lamont \\
\hline Northridge & 1994 & 6.7 & 23.9 & N Faring Rd \\
\hline Northridge & 1994 & 6.7 & 29.5 & N Fletcher Dr \\
\hline Loma Prieta & 1989 & 6.9 & 19.9 & Gilory Array \#6 \\
\hline Loma Prieta & 1989 & 6.9 & 21.4 & Anderson Dam (Downst) \\
\hline Loma Prieta & 1989 & 6.9 & 21.4 & Anderson Dam (Abut) \\
\hline Loma Prieta & 1989 & 6.9 & 22.3 & Coyote Lake Dam \\
\hline Northridge & 1994 & 6.7 & 22.6 & Castaic - Old Ridge Route \\
\hline Northridge & 1994 & 6.7 & 31.3 & LA - Baldwin Hills \\
\hline Northridge & 1994 & 6.7 & 20.8 & Beverly Hills - 12520 Mulhol \\
\hline Northridge & 1994 & 6.7 & 24.0 & Big Tujunga, Angeles Nat F \\
\hline Northridge & 1994 & 6.7 & 25.7 & LA - Century City C C North \\
\hline Northridge & 1994 & 6.7 & 23.7 & LA - Chalon Rd \\
\hline Northridge & 1994 & 6.7 & 17.7 & Sunland - Mt Gleason Ave \\
\hline Northridge & 1994 & 6.7 & 20.0 & Burbank - Howard Rd \\
\hline Northridge & 1994 & 6.7 & 25.7 & Hollywood - Willoughby A \\
\hline Northridge & 1994 & 6.7 & 24.5 & Vasquez Rocks Park \\
\hline San Fernando & 1971 & 6.6 & 24.9 & Castaic - Old Ridge Route \\
\hline
\end{tabular}

Papanikolaou, 2016). As a result, a large amount of earthquake input energy to the building is dissipated due to local degradation in the lateral reinforcement of the structure, so that if it is not taken into consideration, it can ultimately lead to the collapse of the structure.

Figure 3 reveals that using TMD in the studied structure has significantly reduced the story drifts in all first, fifth, tenth, fifteenth and twentieth stories of the structure. This was predictable (Bekdaş and Nigdeli, 2011; Soto and Adeli, 2013) since TMD can prevent structural damages and improve their performance during the earthquakes by absorbing the input energy to the structure (Li and Huo, 2010). According to Figure 3, when using TMD in the structure, the largest relative displacement belongs to the fifteenth story ( $11 \%$ for PGA $\left.=8 \mathrm{~m} \cdot \mathrm{s}^{-2}\right)$, followed by the fifteenth story $\left(10.3 \%\right.$ for $\left.\mathrm{PGA}=9 \mathrm{~m} . \mathrm{s}^{-2}\right)$.

\subsection{Performance of machine learning algorithms}

Results of the prediction of story drifts using supervised models are presented in Table 4. Training and testing performances of each model are shown as MSE and $R^{2}$. Since the MSE values of the models are calculated considering the normalized values of the samples' label, it will be possible to compare the performance of these models. The MSE of the $k$-NN model is presented for $k=$ 1, 2, 3, 4 and 5. The least value of normalized MSE was obtained using the first nearest neighbor (1-NN) model and its values were 0.036 and 0.049 for training and testing, respectively, which are not desirable for accurate prediction of target in geotechnical engineering.

Performance of different types of DT model is also shown in Table 4. Results show that the C4.5 model had acceptable performance as compared with the other DT methods. Although its training MSE was 0.020, the testing performance of this model was greater than 0.01. MSE of 


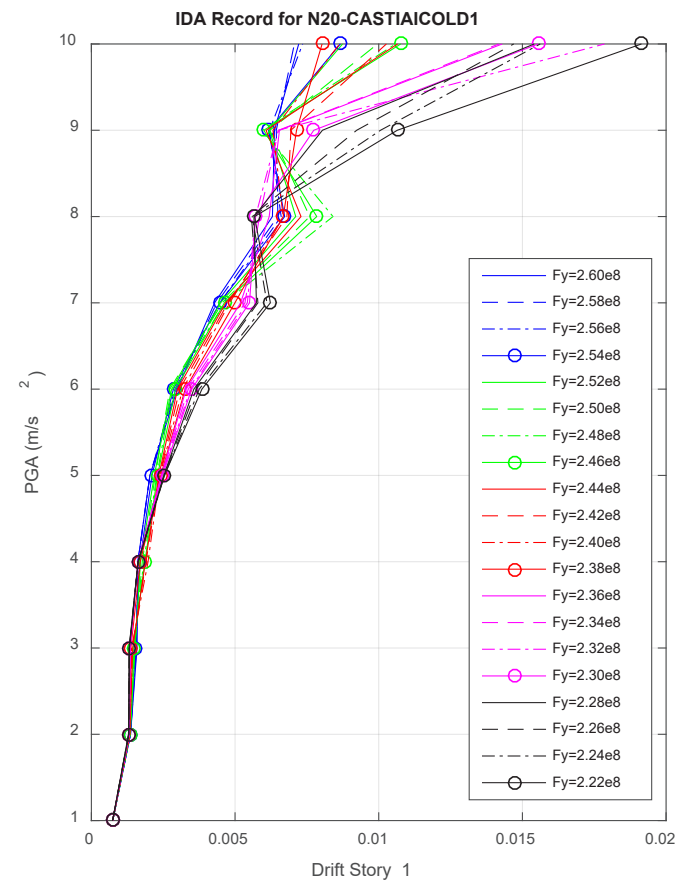

(a)

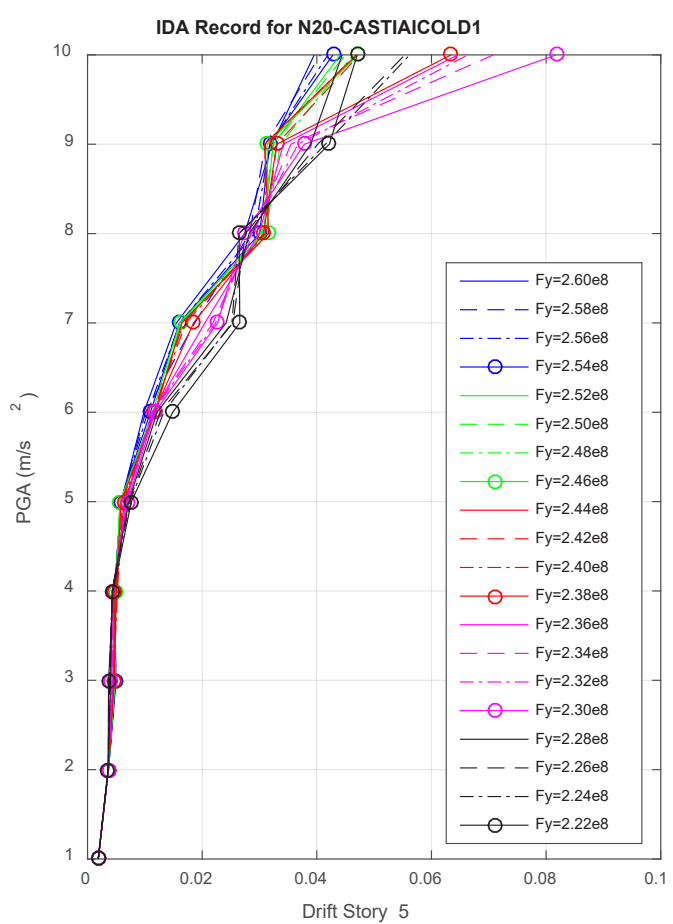

(c)

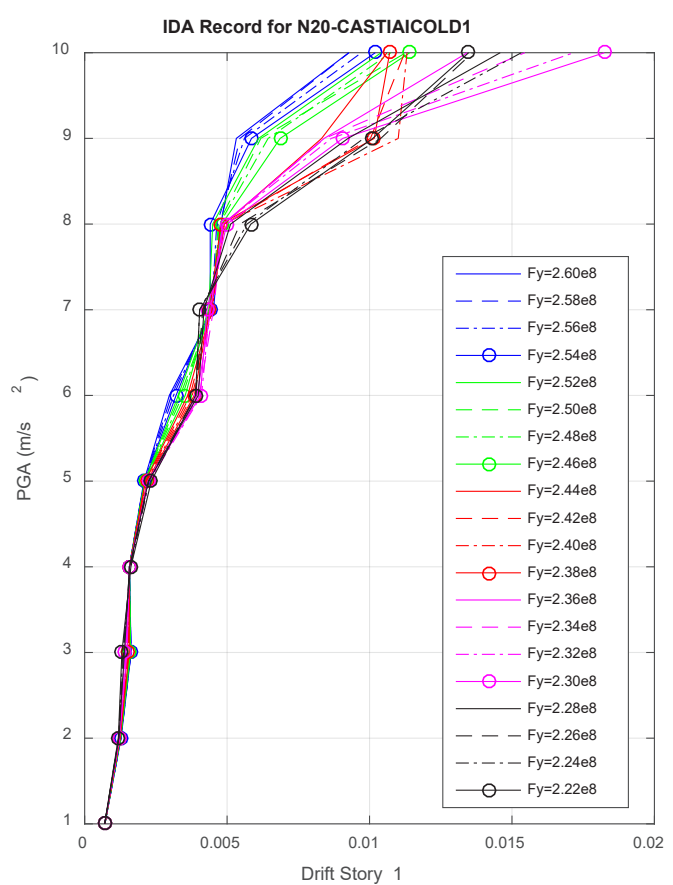

(b)

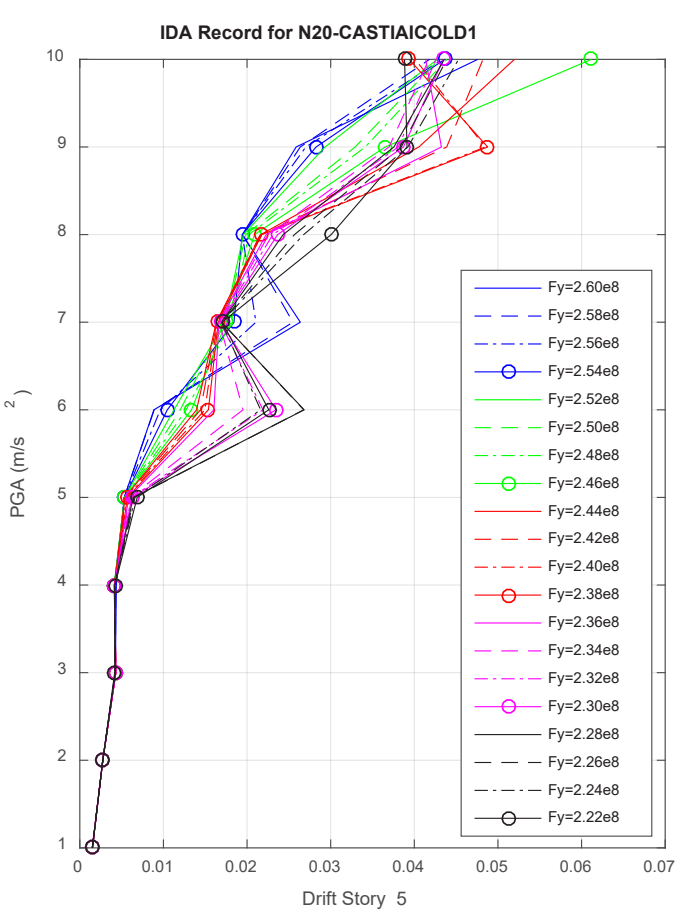

(d)

Figure 3: IDA analysis of the (a,b) first, $(c, d)$ fifth, $(e, f)$ tenth, $(g, h)$ fifteenth, and $(i, j)$ twentieth story of the 20 -story structure (a,c,e,g,i) not equipped with TMD, and (b,d,f,h,j) equipped with TMD 


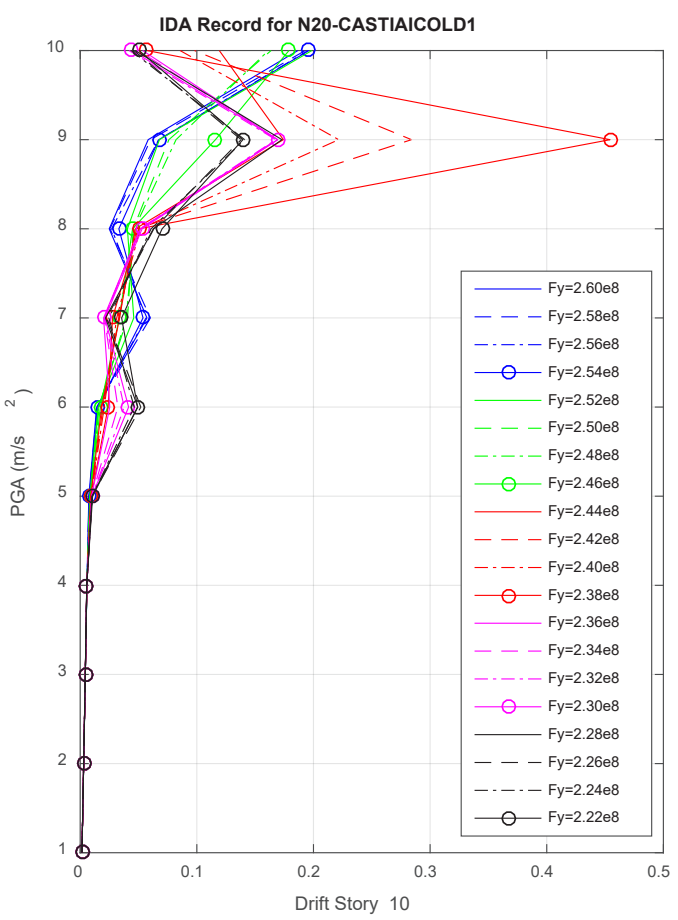

(e)

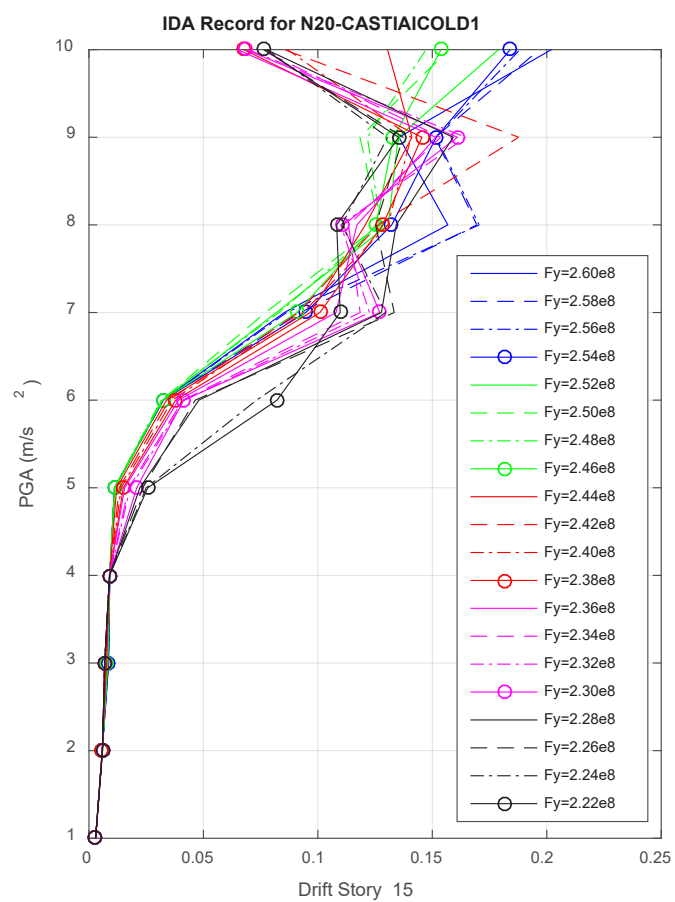

(g)

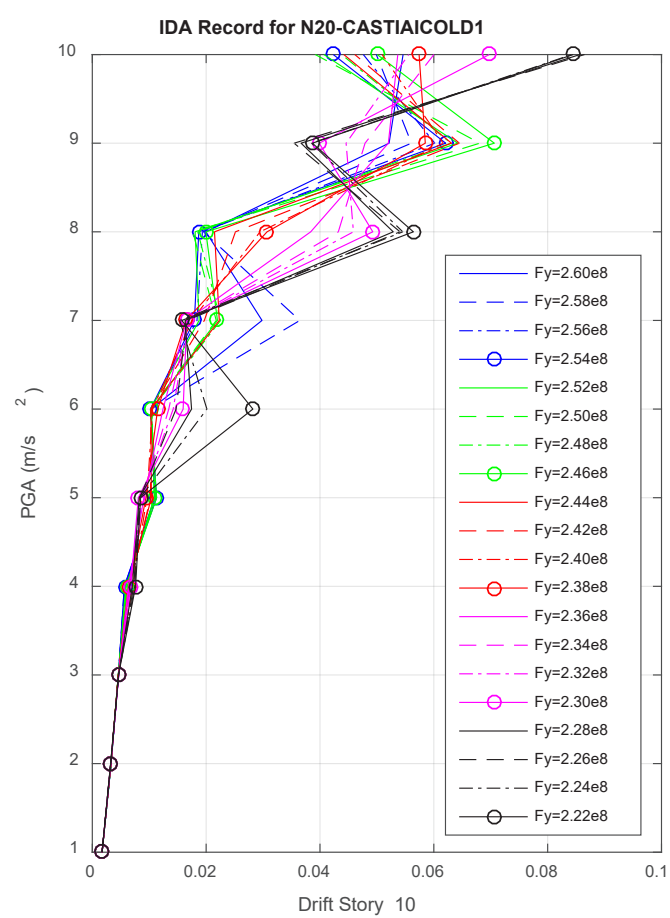

(f)

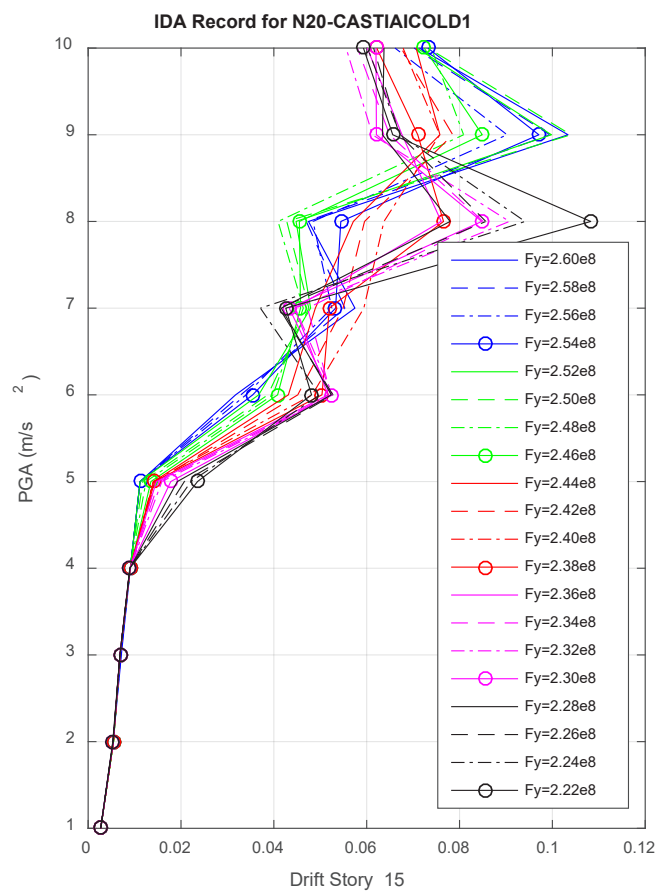

(h)

Figure 3: IDA analysis of the (a,b) first, (c,d) fifth, (e,f) tenth, (g,h) fifteenth, and (i,j) twentieth story of the 20-story structure (a,c,e,g,i) not equipped with TMD, and $(b, d, f, h, j)$ equipped with TMD 


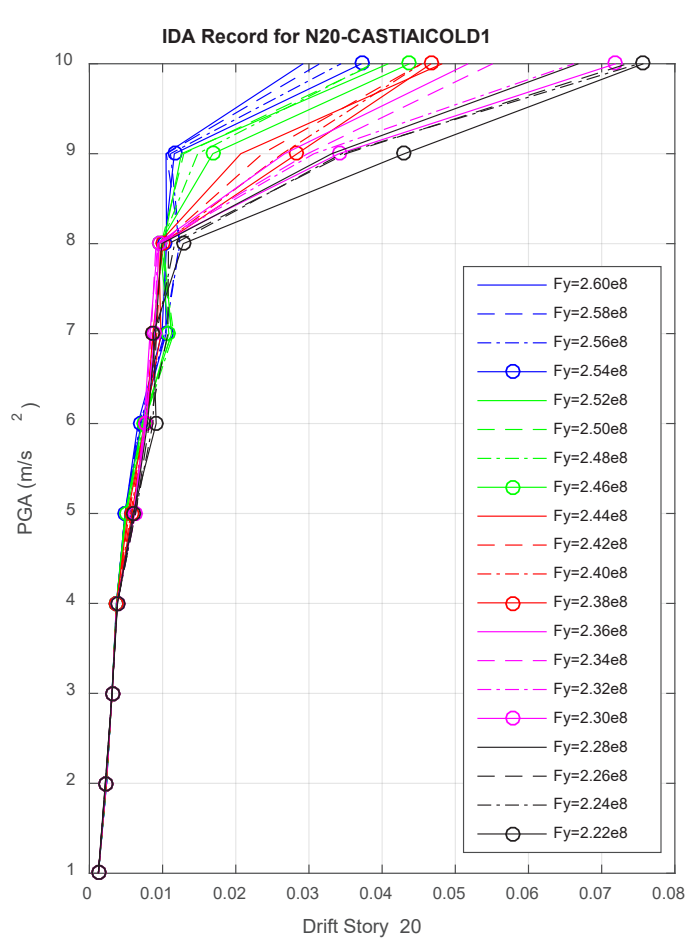

(i)

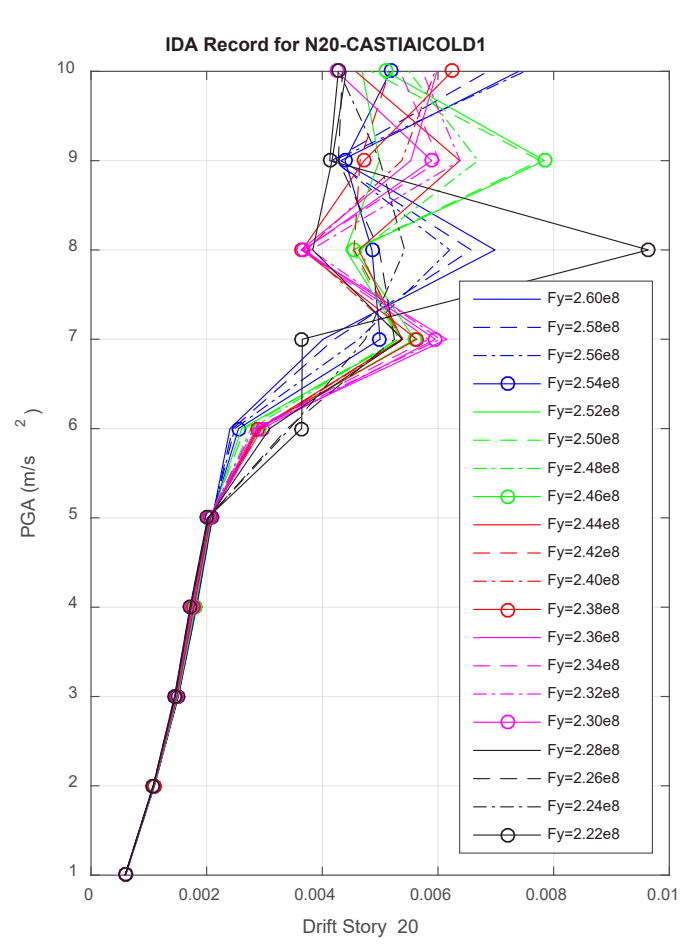

(j)

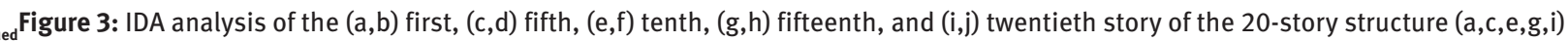
not equipped with TMD, and (b,d,f,h,j) equipped with TMD

$\mathrm{RF}$ model in the prediction of SPR was obtained 0.033 and 0.048 for training and testing, respectively.

One of the important parameters of ANN, which significantly affects its performance, is the network architecture, that is, the size of network hidden layer. In this study, the architecture of the network was 4- $n-1$ (four nodes in the input layer and one node in the output layer). The size of hidden layer varied from 2 to 20 with 2 nodes steps to obtain the optimum network. Results showed that 4-12-1 architecture had the highest performance in comparison with the other network architectures. Study of the performance of different neural network architectures indicates that there is no specific trend between the number of hidden layer neurons and the performance of the network, especially in engineering problems (Hashemi et al., 2014). SVM model with Gaussian kernel and kernel parameter $C=30$ resulted in an acceptable error in regression training and testing. For this type of SVM, training and testing normalized MSE were 0.008 and 0.010 , respectively. These values showed the promising performance of SVM in the prediction of SPR.
Finally, training and testing performance of NB model was obtained as 0.041 and 0.063 , respectively.

Table 4 also shows $R^{2}$ values of the machine learning methods for training and testing procedures. As shown in the table, SVM had the highest $R^{2}$ values for both training and testing procedures. The $R^{2}$ values brought in the table enables us to compare the performance of machine learning models with available statistical models. According to the obtained results, it seems that by using SVM, which is a conventional machine learning model (Massah and Asefpour Vakilian, 2019), geotechnical engineers can simply predict the IDA analysis results having PGA and yield strength at each story of a high steel frame with acceptable performance. In spite of complex and time-consuming methods of IDA analysis, which usually requires several hours to run on available computers, intelligent regression methods can result in proper prediction of drifts in lower than three or four seconds. 
Table 4: Performance evaluation of different models in prediction of the story drifts (parameters of each model are demonstrated in the text).

\begin{tabular}{|c|c|c|c|c|c|}
\hline \multirow{2}{*}{$\begin{array}{l}\text { Regression } \\
\text { model }\end{array}$} & \multirow[t]{2}{*}{ Parameters } & \multicolumn{2}{|c|}{ Normalized MSE } & \multicolumn{2}{|l|}{$R^{2}$} \\
\hline & & Train & Test & Train & Test \\
\hline \multirow[t]{5}{*}{$k-\mathrm{NN}$} & $k=1$ & 0.036 & 0.049 & 0.932 & 0.855 \\
\hline & $k=2$ & 0.036 & 0.050 & 0.932 & 0.849 \\
\hline & $k=3$ & 0.040 & 0.053 & 0.912 & 0.830 \\
\hline & $k=4$ & 0.049 & 0.068 & 0.863 & 0.725 \\
\hline & $k=5$ & 0.045 & 0.066 & 0.886 & 0.740 \\
\hline \multirow[t]{5}{*}{ DT } & type $=$ ID3 & 0.022 & 0.038 & 0.988 & 0.918 \\
\hline & type $=C 4.5$ & 0.020 & 0.033 & 0.994 & 0.942 \\
\hline & type = CART & 0.025 & 0.042 & 0.978 & 0.896 \\
\hline & type $=$ CHAID & 0.043 & 0.060 & 0.897 & 0.783 \\
\hline & type $=$ MARS & 0.049 & 0.056 & 0.863 & 0.810 \\
\hline $\mathrm{RF}$ & - & 0.033 & 0.048 & 0.946 & 0.862 \\
\hline \multirow[t]{10}{*}{ ANN } & hidden layer size $=2$ & 0.050 & 0.066 & 0.857 & 0.740 \\
\hline & hidden layer size $=4$ & 0.040 & 0.059 & 0.912 & 0.790 \\
\hline & hidden layer size $=6$ & 0.039 & 0.054 & 0.917 & 0.824 \\
\hline & hidden layer size $=8$ & 0.046 & 0.070 & 0.880 & 0.710 \\
\hline & hidden layer size $=10$ & 0.039 & 0.052 & 0.917 & 0.837 \\
\hline & hidden layer size $=12$ & 0.030 & 0.049 & 0.959 & 0.855 \\
\hline & hidden layer size $=14$ & 0.037 & 0.058 & 0.927 & 0.797 \\
\hline & hidden layer size $=16$ & 0.042 & 0.061 & 0.902 & 0.776 \\
\hline & hidden layer size $=18$ & 0.051 & 0.067 & 0.851 & 0.732 \\
\hline & hidden layer size $=20$ & 0.050 & 0.058 & 0.857 & 0.797 \\
\hline \multirow[t]{9}{*}{ SVM } & kernel type $=$ linear, $C=10$ & 0.019 & 0.029 & 0.998 & 0.960 \\
\hline & kernel type $=$ linear, $C=20$ & 0.022 & 0.031 & 0.988 & 0.951 \\
\hline & kernel type $=$ linear, $C=30$ & 0.021 & 0.035 & 0.991 & 0.933 \\
\hline & kernel type $=$ Gaussian, $C=10$ & 0.018 & 0.026 & 0.995 & 0.972 \\
\hline & kernel type $=$ Gaussian, $C=20$ & 0.017 & 0.025 & 0.995 & 0.976 \\
\hline & kernel type $=$ Gaussian, $C=30$ & 0.008 & 0.010 & 0.999 & 0.993 \\
\hline & kernel type $=\mathrm{RBF}, C=10$ & 0.030 & 0.038 & 0.959 & 0.918 \\
\hline & kernel type $=\mathrm{RBF}, C=20$ & 0.028 & 0.046 & 0.966 & 0.873 \\
\hline & kernel type $=$ RBF, $C=30$ & 0.029 & 0.046 & 0.962 & 0.873 \\
\hline NB & - & 0.041 & 0.063 & 0.907 & 0.762 \\
\hline
\end{tabular}

\subsection{Results of feature selection}

Table 5 shows the results of sensitivity analysis and feature selection. The scores indicating the importance of structural and seismic uncertainties in predicting the results of the IDA obtained from each feature selection method is given in such a way that the highest score is equal to 1 and the other scores are expressed between 0 and 1. According to this table, the yield strength compared to the maximum acceleration of the earthquake had lower effects on IDA. This suggests that in comparison with the structural uncertainty, seismic uncertainty has a larger contribution to the changes in the results of IDA. It should be noted that the story number parameter also has a significant effect on the drifts, so that predicting IDA only with the maximum acceleration of the earthquake, regardless of the story number in the studied structure leads to a large error in the prediction. The results obtained from different methods of feature selection are similar. For example, Table 5 shows that, in general, the effects of structural and seismic uncertainty in predicting the results of IDA and the story drift on the fifteenth floor of the structure reaches its maximum value, in which the seismic uncertainty has higher effects than structural uncertainty.

Seismic risk for structures has been defined in many ways, ranging from the probability of experiencing a certain ground motion intensity, to the probability of experiencing a certain level of structural response, to the probability of experiencing failure in a critical failure mode, to the probability of system failure which is the ultimate consequence of failure in a number of 
Table 5: Results of feature selection for sensitivity analysis of seismic and structural uncertainties.

\begin{tabular}{lllllllll}
\hline Story & \multicolumn{2}{l}{ Seismic uncertainty } & \multicolumn{5}{l}{ Structural uncertainty } \\
\cline { 2 - 8 } & Relieff & t-test & SFS & CSC & Relieff & t-test & SFS & CSC \\
\hline 1 & 0.44 & 0.51 & 0.50 & 0.68 & 0.23 & 0.30 & 0.29 & 0.11 \\
5 & 0.53 & 0.60 & 0.52 & 0.69 & 0.44 & 0.35 & 0.45 & 0.32 \\
10 & 0.85 & 1.00 & 0.76 & 0.82 & 0.70 & 0.59 & 0.71 & 0.77 \\
15 & 1.00 & 0.95 & 1.00 & 1.00 & 0.73 & 0.86 & 0.80 & 0.83 \\
20 & 0.84 & 0.81 & 0.79 & 0.78 & 0.70 & 0.79 & 0.74 & 0.79 \\
\hline
\end{tabular}

interdependent subsystems comprising a functional system. Although Table 5 reveals that seismic uncertainty can be more effective on IDA analysis results compared with structural uncertainty, the table shows that structural uncertainty is also a matter of great importance in accurate behavior analysis of high steel frames and geotechnical and earthquake engineers should always consider this type of uncertainty during their designings.

\section{Conclusions}

In this study, the probability of failure of steel structures equipped with TMD is firstly investigated considering seismic and structural uncertainties and then, the performance of several machine learning algorithms is investigated in the prediction of the structure's behavior. For this purpose, a 20-story steel structure was modeled, and then the results of IDA for several seismic records were studied. Results of this study show that SVM with Gaussian kernel has better performance since it is capable of predicting the drift of stories of the studied structure with $R^{2}$ value equal to 0.99 . Furthermore, results of feature selection algorithms reveal that when using TMD in high steel structures, seismic uncertainties have greater influences on drift of stories in comparison with structural uncertainties.

\section{References}

[1] Ahmad, M. F., Haydar, S., Bhatti, A. A., Bari, A. J. (2014). Application of artificial neural network for the prediction of biosorption capacity of immobilized Bacillus subtilis for the removal of cadmium ions from aqueous solution. Biochemical engineering journal, 84, 83-90.

[2] Asefpour Vakilian, K., Massah, J. (2018). A portable nitrate biosensing device using electrochemistry and spectroscopy. IEEE Sensors Journal, 18(8), 3080-3089.
[3] Basak, D., Pal, S., Patranabis, D. C. (2007). Support vector regression. Neural Information Processing-Letters and Reviews, 11(10), 203-224.

[4] Bekdaş, G., Nigdeli, S. M. (2011). Estimating optimum parameters of tuned mass dampers using harmony search. Engineering Structures, 33(9), 2716-2723.

[5] Chey, M. H., Chase, J. G., Mander, J. B., Carr, A. J. (2010). Semi-active tuned mass damper building systems: Application. Earthquake Engineering \& Structural Dynamics, 39(1), 69-89.

[6] Cramér H. (2016). Mathematical Methods of Statistics, vol. 9. Princeton, NJ, USA: Princeton University Press.

[7] Debbarma R., Debnath D. (2013). Earthquake Response Control of 3-Story Building Structures by Tuned Mass Damper. International Journal of Engineering and Innovative Technology, 2, 187-192.

[8] Deierlein, G. G., Reinhorn, A. M., Willford, M. R. (2010). Nonlinear structural analysis for seismic design. NEHRP seismic design technical brief, 4, 1-36.

[9] Farrokhi, F., Rahimi, S. (2017). Probabilistic failure analysis of high steel frames with tuned mass damper. In XI Conference on Steel and Composite Construction, Coimbra, Portugal, 23 and 24 November, 2017, 507-514.

[10] Giaralis, A., Taflanidis, A. A. (2018). Optimal tuned massdamper-inerter (TMDI) design for seismically excited MDOF structures with model uncertainties based on reliability criteria. Structural Control and Health Monitoring, 25(2), e2082.

[11] Guyon, I., Elisseeff, A. (2003). An introduction to variable and feature selection. Journal of machine learning research, 3 , 1157-1182.

[12] Hashemi, A., Asefpour Vakilian, K., Khazaei, J., Massah, J. (2014). An artificial neural network modeling for force control system of a robotic pruning machine. Journal of Information and Organizational Sciences, 38(1), 35-41.

[13] Huang, J., Cai, Y., Xu, X. (2007). A hybrid genetic algorithm for feature selection wrapper based on mutual information. Pattern Recognition Letters, 28(13), 1825-1844.

[14] Kappos, A. J., Papanikolaou, V. K. (2016). Nonlinear dynamic analysis of masonry buildings and definition of seismic damage states. The Open Construction and Building Technology Journal, 10(1), 192-209.

[15] Krishnan, S. (2007). Case studies of damage to 19-storey irregular steel moment-frame buildings under near-source ground motion. Earthquake engineering \& structural dynamics, 36(7), 861-885. 
[16] Li, H., Huo, L. (2010). Advances in structural control in civil engineering in China. Mathematical Problems in Engineering, 1-23.

[17] Lin, S. W., Lee, Z. J., Chen, S. C., Tseng, T. Y. (2008). Parameter determination of support vector machine and feature selection using simulated annealing approach. Applied soft computing, 8(4), 1505-1512.

[18] Liu, H., Li, J., Wong, L. (2002). A comparative study on feature selection and classification methods using gene expression profiles and proteomic patterns. Genome informatics, 13 , 51-60.

[19] Liu, H., Liu, L., Zhang, H. (2009). Boosting feature selection using information metric for classification. Neurocomputing, 73(1), 295-303.

[20] Marano, G. C., Greco, R., Chiaia, B. (2010). A comparison between different optimization criteria for tuned mass dampers design. Journal of Sound and Vibration, 329(23), 4880-4890.

[21] Massah, J., Asefpour Vakilian, K. (2019). An intelligent portable biosensor for fast and accurate nitrate determination using cyclic voltammetry. Biosystems Engineering, 177, 49-58.

[22] Michalski, R.S., Carbonell, J. G., Mitchell, T. M. (2013). Machine Learning, An Artificial Intelligence Approach. Berlin, Germany: Springer.

[23] Mohebbi, M., Shakeri, K., Ghanbarpour, Y., Majzoub, H. (2013). Designing optimal multiple tuned mass dampers using genetic algorithms (GAs) for mitigating the seismic response of structures. Journal of Vibration and Control, 19(4), 605-625.

[24] Moustakidis, S. P., Theocharis, J. B. (2010). SVM-FuzCoC: A novel SVM-based feature selection method using a fuzzy complementary criterion. Pattern Recognition, 43(11), 37123729.

[25] Muto, M., Krishnan, S. (2011). Hope for the best, prepare for the worst: Response of tall steel buildings to the shakeout scenario earthquake. Earthquake Spectra, 27(2), 375-398.

[26] Pozos-Estrada, A., Hong, H. P. (2015). Sensitivity Analysis of the Effectiveness of Tuned Mass Dampers to Reduce the WindInduced Torsional Responses. Latin American Journal of Solids and Structures, 12(13), 2520-2538.

[27] Sadek, F., Mohraz, B., Taylor, A. W., Chung, R. M. (1997). A method of estimating the parameters of tuned mass dampers for seismic applications. Earthquake Engineering \& Structural Dynamics, 26(6), 617-635.

[28] Soto, M. G., Adeli, H. (2013). Tuned mass dampers. Archives of Computational Methods in Engineering, 20(4), 419-431.

[29] Sun, X., Liu, Y., Li, J., Zhu, J., Chen, H., Liu, X. (2012). Feature evaluation and selection with cooperative game theory. Pattern recognition, 45(8), 2992-3002.

[30] Vickery, B. J., Galsworthy, J. K., Gerges, R. (2001). The behaviour of simple non-linear tuned mass dampers. In 6th World Congress of the Council on Tall Buildings and Urban Habitat, Melbourne, Australia.

[31] Wang, L., Shi, W., Zhou, Y. (2019). Study on self-adjustable variable pendulum tuned mass damper. The Structural Design of Tall and Special Buildings, 28(1), e1561. 